Pacific Journal of Mathematics

ON THE KATO-ROSENBLUM THEOREM 


\title{
ON THE KATO-ROSENBLUM THEOREM
}

\author{
JAMES S. HOWLAND
}

The Kato-Rosenblum Theorem has no straightforward generalization to operators with non-absolutely continuous spectra. For example, if $A$ is a bounded selfadjoint operator such that the singular continuous parts of $H$ and $H+A$ are unitarily equivalent for every selfadjoint operator $H$, then $A=0$.

1. Introduction. The classical theorem of Kato and Rosenblum (1957) asserts the invariance of absolutely continuous parts under tracse class perturbations. [5, p. 540; 6, p. 26]

TheOREM (Kato-Rosenblum). If $H$ and $A$ are selfadjoint, and $A$ is trace class, then the absolutely continuous parts of $H$ and $H+A$ are unitarily equivalent.

It is notable that the theorem gives a unitarily invariant condition on the perturbation $A$ alone, and that Lebesgue measure plays a distinguished role.

That the trace condition cannot be radically improved, follows from the Weyl-von Neumann theorem [5, p. 523], which states that given any selfadjoint operator $H$, there is a selfadjoint perturbation $A$ of arbitrarily small Hilbert-Schmidt norm, such that $H+A$ has pure point spectrum-a phenomenon often termed curdling. Moreover, according to Kuroda, the Hilbert-Schmidt norm may be replaced by any cross-norm except the trace norm. [5, p. 525]

For singular measures, there are a few, largely negative, results. Donoghue [2], following earlier work of Aronszajn, gave examples in which a purely singular continuous spectrum is curdled by a perturbation of rank one. He also obtained the following result, which we shall use [ $\mathbf{2}$, p. $565 ; 4$, Cor. 1$]$.

Theorem. (Donoghue). Let $H$ be selfadjoint and $A=c\langle\cdot, \phi\rangle \phi$ where $\phi$ if cyclic for $H$ and $c$ is real and non-zero. Then the singular parts of $H$ and $H+A$ are supported on disjoint sets (i.e. are mutually singular).

A generalization was proved in [4]. 
Following Donoghue's approach, Carey and Pincus [1] proved that the spectrum of any operator with purely singular spectrum can be curdled by a perturbation of arbitrarily small trace norm. A proof of this fact following the Weyl-von Neumann construction has recently been given by Eugene Wayne [6].

These results leave it difficult to imagine a unitarily invariant condition on $A$ alone which might guarantee that $A$ preserves singular continuous parts. Indeed, as we shall prove, there is no such condition: if $H$ and $H+A$ have unitarily equivalent singular parts for every $H$, then $A=0$.

We shall, in fact, prove that it is impossible to generalize the KatoRosenblum theorem to other measures in the following sense. Let $\mu$ be a non-zero Borel measure, and $A \neq 0$ a bounded operator. If the parts of $H$ and $H+A$ which are absolutely continuous with respect to $\mu$ are unitarily equivalent for all selfadjoint $H$, then $\mu$ is absolutely continuous with respect to Lebesgue measure, and, moreover, the entire absolutely continuous parts of $H$ and $H+A$ are unitarily equivalent.

We shall also prove that $A$ is necessarily compact. The Weyl-von Neumann-Kuroda result strongly suggests that $A$ is trace class, but we know of no proof.

The author wishes to thank Ira Herbst and Eugene Wayne for valuable conversations. He has, however, resisted Professor Herbst's rather gratuitous suggestion that this paper be entitled "A New Characterization of Lebesgue Measure."

2. Preservation of measures. Let $\mathscr{H}$ be a separable Hilbert space, and $H=\int \lambda E(d \lambda)$ a selfadjoint operator on $\mathscr{H}$. We shall assume throughout that all operators are bounded. For Borel measures $m$ and $\mu$ on $\mathbf{R}$, write $u \ll \mu$ if $m$ is absolutely continuous with respect to $\mu$. For $x \in \mathscr{H}$, let $m_{x}$ be the Borel measure

$$
m_{x}(d \lambda)=\langle E(d \lambda) x, x\rangle \text {. }
$$

The set

$$
\mathscr{H}_{\mu}(H)=\left\{x \in \mathscr{H}: m_{x} \ll \mu\right\}
$$

is a closed reducing subspace of $H$, called the absolutely continuous subspace of $H$ with respect to $\mu$. Its orthogonal complement is

$$
\mathscr{H}_{\mu}^{s}(H)=\left\{x \in \mathscr{H}: m_{x} \text { and } \mu \text { are mutually singular }\right\} .
$$

(See [5, p. 516.] The proof is given for Lebesgue measure, but holds in general without change.) 
For any Borel measure $\mu$, define $H_{\mu}$ to be the restriction of $H$ to $\mathscr{H}_{\mu}(H)$. If $\nu \ll \mu$, then

$$
\left(H_{\mu}\right)_{\nu}=H_{\nu} \text {. }
$$

For real $t$, define the translated measure

$$
\mu_{t}(S)=\mu(S-t)
$$

Then

$$
(H+t)_{\mu_{t}}=H_{\mu}+t .
$$

Write $A \cong B$ to mean that $A$ and $B$ are unitarily equivalent.

2.1 Definition. Let $\mu$ be a Borel measure on $\mathbf{R}$. A selfadjoint operator A preserves $\mu$ iff

$$
(H+A)_{\mu} \cong H_{\mu}
$$

for every selfadjoint operator $H$.

The trivial zero measure is preserved by every $A$, because the space $\mathscr{H}_{\mu}$ is then always zero-dimensional. The Kato-Rosenblum theorem says that trace class operators preserve Lebesgue measure.

2.2 Proposition. Let $A$ and $B$ preserve $\mu$. Then:

(a) $A+B$ and $c A$ also preserves $\mu$, if $c$ is real:

(b) if $\nu \ll \mu$, then $A$ preserves $\nu$;

(c) $A$ preserves $\mu_{t}$ for all $t$;

(d) if $W \cong A$, then $W$ preserves $\mu$; and

(e) If $P$ is an orthogonal reducing projection for $A$, then AP preserves $\mu$ on $P \mathscr{H}$;

Proof.

(a) We have

$$
(H+A+B)_{\mu} \cong(H+A)_{\mu} \cong H_{\mu} .
$$

and similarly

$$
(H+c A)_{\mu}=c\left(c^{-1} H+A\right)_{\mu} \cong c\left(c^{-1} H\right)_{\mu}=H_{\mu} .
$$

(b) By (2.1),

$$
(H+A)_{\nu}=\left[(H+A)_{\mu}\right]_{\nu} \cong\left(H_{\mu}\right)_{\nu}=H_{\nu} .
$$

(c) By (2.2),

$$
(H+A)_{\mu_{t}}=(H+t+A)_{\mu}-t \cong(H+t)_{\mu}-t=H_{\mu_{t}} .
$$


(d) If $W=U A U^{*}$, with $U$ unitary, then

$$
\begin{aligned}
(H+W)_{\mu} & =\left(H+U A U^{*}\right)_{\mu}=\left[U\left(U^{*} H U+A\right) U^{*}\right]_{\mu} \\
& \cong\left(U^{*} H A U+A\right)_{\mu} \cong\left(U^{*} H U\right)_{\mu} \cong H_{\mu}
\end{aligned}
$$

(e) Let $A$ be the restriction of $A$ of $P \mathscr{H}$. Writing operator matices for the decomposition $\mathscr{H}=P \mathscr{H} \oplus(I-P) \mathscr{H}$ gives

$$
A=\left(\begin{array}{cc}
A_{1} & 0 \\
0 & A_{2}
\end{array}\right)
$$

If $H$ is defined on $\mathscr{H}$ by

$$
H=\left(\begin{array}{cc}
H_{1} & 0 \\
0 & 0
\end{array}\right)
$$

then $(H+A)_{\mu} \cong H_{\mu}$ says that

$$
\left(\begin{array}{cc}
\left(H_{1}+A_{1}\right)_{\mu} & 0 \\
0 & \left(A_{2}\right)_{\mu}
\end{array}\right) \cong\left(\begin{array}{cc}
\left(H_{1}\right)_{\mu} & 0 \\
0 & 0_{\mu}
\end{array}\right)
$$

which gives $\left(H_{1}+A_{1}\right)_{\mu} \cong\left(H_{1}\right)_{\mu}$, by equating the first components.

TheOREM 1. If $A$ preserves a non-zero measure $\mu$, then $A$ is compact.

Proof. If $A$ is not compact, then, possibly replacing $A$ by $-A$, there is an infinite dimensional reducing projection $P$ of $A$ such that $A_{1}=A P \geq$ $\delta P$ for some $\delta>0$. By restriction and translation (2.2(b) and (c)), we can assume that $[0,1]$ supports $\mu$, and that $\mu[0, \varepsilon]>0$ for every $\varepsilon>0$. Choosing $H_{1}$ to be an operator on $P \mathscr{H}$ unitarily equivalent to multiplication by $\lambda$ on $\mathscr{L}^{2}([0,1], d \mu(\lambda))$, we see that $H_{1} \geq 0$ and that the spectrum of $H_{1}=\left(H_{1}\right)_{\mu}$ contains 0 . By $(\mathrm{e})$,

$$
\left(H_{1}+A_{1}\right)_{\mu}=\left(H_{1}\right)_{\mu} \text {. }
$$

But $H_{1}+A_{1} \geq \delta P>0$, so 0 is not in the spectrum of $H_{1}+A_{1}$, a contradiction. Hence, $A$ is compact.

Theorem 2. If $A$ preserves $\mu$ and $A \neq 0$, then $\mu$ is absolutely continuous with respect to Lebesgue measure.

Proof. Choose a vector $\phi$ of norm one, which is not an eigenvector of $A$, but for which $A \phi \neq 0$. The operator

$$
U=1-2 \cdot\langle\cdot, \phi\rangle \phi
$$

is unitary, and we compute that

$$
B=A-U A U^{*}=2\langle\cdot, A \phi\rangle \phi+2\langle\cdot, \phi\rangle A-4\langle A \phi, \phi\rangle\langle\cdot, \phi\rangle \phi .
$$


Since $\phi$ and $A \phi$ are independent, $B$ has rank exactly two. By 2.2(a) and (d), $B$ also preserves $\mu$.

Let $\phi_{1}$ and $\phi_{2}$ be the two eigenvectors of $B$ with non-zero eigenvalues. Let $\mathscr{H}_{1}$ be the orthogonal complement of $\phi_{1}$, which reduces $B$. By 2.1(e), the restriction $B_{1}$ of $B$ to $\mathscr{H}_{1}$ must preserve $\mu$ on $\mathscr{H}_{1}$. But $B_{1}=c\left\langle\cdot, \phi_{2}\right\rangle \phi_{2}$ has rank one. Since $H_{\mu}$ depends only on a class of mutually absolutely continuous measures, we can assume that $\mu$ is finite, with total mass one. Identify $\mathscr{H}_{1}$ with $L^{2}(\mu)$ and $\phi_{2}$ with the constant function 1 , and let $H_{1}$ be multiplication by $\lambda$. Since $\phi_{2}$ is cyclic for $H_{1}$, Donoghue's theorem says that the singular parts of $H_{1}$ and $H_{1}+B_{1}=H_{1}$ $+c\left\langle\cdot, \phi_{2}\right\rangle \phi_{2}$ are disjointly supported. Thus, if $\mu$ had a singular part, $B_{1}$ could not preserve $\mu$, so $\mu$ must be absolutely continuous.

TheOREM 3. If $A$ preserves a non-zero absolutely continuous measure, then $A$ preserves Lebesgue measure.

Denote by $\chi_{S}(\lambda)$ the characteristic function of the Borel set $S$, by $|S|$ its Lebesgue measure, and by $\mu_{S}$, the measure

$$
\mu_{S}(d \lambda)=\chi_{S}(\lambda) d \lambda
$$

Write $H_{S}$ for $H_{\mu_{S}}$. Let

$$
\mathscr{B}=\left\{S: A \text { preserves } \mu_{S}, S \text { Borel }\right\} .
$$

2.3 LEMMA. (i) $\mathscr{B}$ contains every set of measure zero.

(ii) $\mathscr{B}$ contains a set of positive measure.

(iii) If $S \in \mathscr{B}$, then $S+t \in \mathscr{B}$ for every $t$

(iv) If $S \in \mathscr{B}$ and $F \subset S$, then $F \in \mathscr{B}$. Hence, $\mathscr{B}$ is closed under intersection and difference.

(v) $\mathscr{B}$ is closed under countable unions.

Proof. (i) If $|S|=0, \mu_{S}$ is the zero measure, which is always preserved.

(ii) If $A$ preserves the measure $f(\lambda) d \lambda$, with density $f(\lambda)$, then $S=\{\lambda: f(\lambda)>0\}$ is in $\mathscr{B}$ and has positive measure.

(iii) follows from 2.2(c), and (iv) from 2.2(b).

Writing

(v) Let $S=S_{1} \cup S_{1} \cup \cdots$, with $S_{j} \in \mathscr{B}$.

$$
S=S_{1} \cup\left(S_{2} \cup S_{1}\right) \cup\left(S_{2} \sim\left[S_{1} \cup S_{2}\right]\right) \cup \cdots
$$


and noting (iv) permits us to assume that $S_{1}, S_{2} \ldots$ are disjoint. In that case

$$
(H+A)_{S} \cong \bigoplus \sum_{j \geq 1}(H+A)_{S_{J}} \cong \bigoplus \sum_{j \geq 1} H_{S_{J}} \cong H_{S}
$$

so that $S \in \mathscr{B}$.

Proof of Theorem 3. We wish to show that $\mathscr{B}$ contains the whole line R. By (iii) and (v), it suffices that $\mathscr{B}$ contain $[0,1]$. Let

$$
\mathscr{B}_{0}=\{S \in \mathscr{B}: S \subset[0,1]\} .
$$

If we can prove that

$$
\sup \left\{|S|: S \in \mathscr{B}_{0}\right\}=1
$$

then (cf. [3, p. 75]) the union $F$ of a sequence of sets $F_{n} \in \mathscr{B}_{0}$ with $\left|F_{\mu}\right| \rightarrow 1$, is in $\mathscr{B}_{0}$ and has measure 1 . Hence $[0,1]$, which is the union of $F$ with a null set, is also in $\mathscr{B}_{0}$.

It remains to prove (2.3). Let $\varepsilon>0$ and $0<\alpha<1$ be arbitrary. Use (i), (ii) and (iii) to find an $S \in \mathscr{B}_{0}$ with $0<|S|<\varepsilon$, and then an interval $I$ with

$$
|I \cap S|>\alpha|I|
$$

[3, p. 68]. Note that $|I|<\varepsilon / \alpha$.

Lay off on $[0,1]$ consecutive intervals $I_{1}, I_{2}, \ldots$ of the same length as $I$, starting at 0 and continuing until $I_{1}, \ldots, I_{n+1}$ just cover $[0,1]$. Each $I_{j}$ is a translate $I_{j}=I+t_{j}$ of $I$. If $F_{j}=(I \cap S)+t_{j}$, and $F=F_{1}$ $\cup \cdots \cup F_{n}$, then $F, F_{J} \in \mathscr{B}_{0}$ and

$$
\begin{aligned}
|F| & =\left|F_{1} \cup \cdots \cup F_{n}\right|=N|I \cap S|>N \alpha|I| \\
& =\alpha\left|I_{1} \cup \cdots \cup I_{n}\right|>\left(1-\left|I_{n+1}\right|\right)>\alpha(1-\varepsilon / \alpha) .
\end{aligned}
$$

The right side can be made arbitrarily close to 1 by choosing $\varepsilon$ small and $\alpha$ close to 1 .

\section{REFERENCES}

[1] R. W. Carey and J. D. Pincus, Unitary equivalence modulo the trace class for selfadjoint operators, Amer. J. Math., 98 (1976), 481-514.

[2] W. F. Donoghue, On the perturbation of spectra, Comm. Pure Appl. Math., 18 (1965), 559-579.

[3] P. R. Holmes, Measure Theory, Van Nostrand, Princeton, N. J., 1950. 
[4] J. S. Howland, On a theorem of Aronszajn and Donaghue on singular spectra, Duke J. Math., 41 (1974), 141-143.

[5] T. Kato, Perturbation Theory for Linear Operators, Springer-Verlag, New York, 1966.

[6] M. Reed and B. Simon, Methods of Modern Mathematical Physics, v. III. Academic Press, New York, 1979.

[7] E. Wayne, private communication.

Received March 8, 1985. Supported by MCS-82-02115-01

UNIVERSITY OF VIRGINIA

Charlottesville, VA 22903-3199 



\title{
PACIFIC JOURNAL OF MATHEMATICS EDITORS
}

\author{
V. S. VARADARAJAN \\ (Managing Editor) \\ University of California \\ Los Angeles, CA 90024 \\ HERBERT ClEMENS \\ University of Utah \\ Salt Lake City, UT 84112 \\ R. FINN \\ Stanford University \\ Stanford, CA 94305
}

\author{
HERMANN FLASCHKA \\ University of Arizona \\ Tucson, AZ 85721 \\ RAMESH A. GANGOLLI \\ University of Washington \\ Seattle, WA 98195 \\ VAUGHAN F. R. JONES \\ University of California \\ Berkeley, CA 94720 \\ ROBION KIRBY \\ University of California \\ Berkeley, CA 94720
}

C. C. MOORE

University of California

Berkeley, CA 94720

H. SAMELSON

Stanford University

Stanford, CA 94305

HAROLD STARK

University of California, San Diego

La Jolla, CA 92093

\section{ASSOCIATE EDITORS}

\author{
R. AREnS \\ E. F. BECKENBACH \\ B. H. NEUMANN \\ F. WOLF \\ K. YOSHIDA \\ (1906-1982)

\section{SUPPORTING INSTITUTIONS}

\begin{abstract}
UNIVERSITY OF ARIZONA
UNIVERSITY OF BRITISH COLUMBIA

UNIVERSITY OF CALIFORNIA

MONTANA STATE UNIVERSITY

UNIVERSITY OF NEVADA, RENO

NEW MEXICO STATE UNIVERSITY

OREGON STATE UNIVERSITY
\end{abstract} \\ CALIFORNIA INSTITUTE OF TECHNOLOGY \\ UNIVERSITY OF OREGON \\ UNIVERSITY OF SOUTHERN CALIFORNIA \\ STANFORD UNIVERSITY \\ UNIVERSITY OF HAWAII \\ UNIVERSITY OF TOKYO \\ UNIVERSITY OF UTAH \\ WASHINGTON STATE UNIVERSITY \\ UNIVERSITY OF WASHINGTON
}

The Supporting Institutions listed above contribute to the cost of publication of this Journal, but they are not owners or publishers and have no responsibility for its content or policies.

Mathematical papers intended for publication in the Pacific Journal of Mathematics should be in typed form or offset-reproduced (not dittoed), double spaced with large margins. Please do not use built up fractions in the text of the manuscript. However, you may use them in the displayed equations. Underline Greek letters in red, German in green, and script in blue. The first paragraph must be capable of being used separately as a synopsis of the entire paper. In particular it should contain no bibliographic references. Please propose a heading for the odd numbered pages of less than 35 characters. Manuscripts, in triplicate, may be sent to any one of the editors. Please classify according to the scheme of Math. Reviews, Index to Vol. 39. Supply name and address of author to whom proofs should be sent. All other communications should be addressed to the managing editor, or Elaine Barth, University of California, Los Angeles, California 90024.

There are page-charges associated with articles appearing in the Pacific Journal of Mathematics. These charges are expected to be paid by the author's University, Government Agency or Company. If the author or authors do not have access to such Institutional support these charges are waived. Single authors will receive 50 free reprints; joint authors will receive a total of 100 free reprints. Additional copies may be obtained at cost in multiples of 50 .

The Pacific Journal of Mathematics is issued monthly as of January 1966. Regular subscription rate: $\$ 190.00$ a year (5 Vols., 10 issues). Special rate: $\$ 95.00$ a year to individual members of supporting institutions.

Subscriptions, orders for numbers issued in the last three calendar years, and changes of address should be sent to Pacific Journal of Mathematics, P.O. Box 969, Carmel Valley, CA 93924, U.S.A. Old back numbers obtainable from Kraus Periodicals Co., Route 100, Millwood, NY 10546.

The Pacific Journal of Mathematics at P.O. Box 969, Carmel Valley, CA 93924 (ISSN 0030-8730) publishes 5 volumes per year. Application to mail at Second-class postage rates is pending at Carmel Valley, California, and additional mailing offices. Postmaster: send address changes to Pacific Journal of Mathematics, P.O. Box 969, Carmel Valley, CA 93924.

PUBLISHED BY PACIFIC JOURNAL OF MATHEMATICS, A NON-PROFIT CORPORATION

Copyright (C) 1986 by Pacific Journal of Mathematics 


\section{Pacific Journal of Mathematics}

\section{Vol. 123, No. $2 \quad$ April, 1986}

David Jay Anick, A loop space whose homology has torsion of all orders . . 257 Steven P. Diaz, Space curves that intersect often ................. 263

Thierry Fack and Hideki Kosaki, Generalized $s$-numbers of $\tau$-measurable

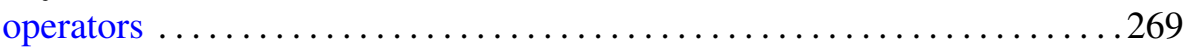

Karl Heinrich Hofmann and Karl Strambach, Lie's fundamental

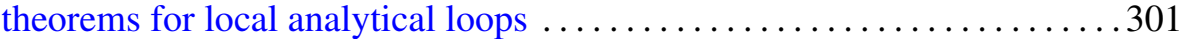

James Secord Howland, On the Kato-Rosenblum theorem ............. 329

Frieder Knüppel and Edzard Salow, Plane elliptic geometry over rings . . . . 337

Alan Noell, Peak points in boundaries not of finite type ................ 385

William J. Ralph, An extension of singular homology to Banach

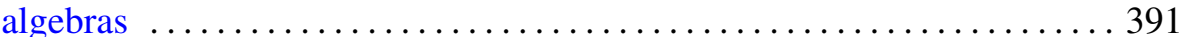

Wade C. Ramey, Averaging properties of pluriharmonic boundary values . . 407

Thomas Joseph Ransford, On the range of an analytic multivalued

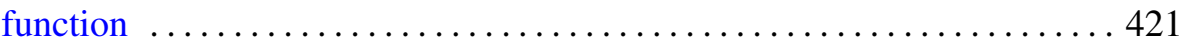

Christopher Donald Sogge, On restriction theorems of maximal-type . . . . 441

Edwin Spanier, Cohomology with supports $\ldots \ldots \ldots \ldots \ldots \ldots \ldots \ldots \ldots 47$

Emil J. Straube, Orthogonal projections onto subspaces of the harmonic

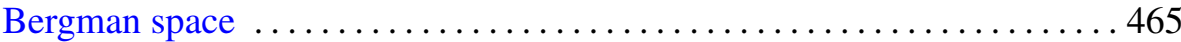

Thomas Vogel, Asymptotic behavior of two semilinear elliptic free boundary problems $\ldots \ldots \ldots \ldots \ldots \ldots \ldots \ldots \ldots$ 